\title{
Object wave reconstruction by speckle illumination and phase retrieval
}

\author{
P. F. Almoro \\ percival.almoro@risoe.dk
}

\section{S. G. Hanson}

\author{
DTU Fotonik, Department of Photonics Engineering, Danish Technical University, \\ Roskilde 4000, Denmark \\ DTU Fotonik, Department of Photonics Engineering, Danish Technical University, \\ Roskilde 4000, Denmark
}

An innovative setup for the speckle-based phase retrieval method is proposed. In the conventional setup, a plane wave illuminates the test object and the transmitted wavefront is incident on a diffuser aperture generating a speckle field. The sampled speckle intensities at axially displaced planes are input into a phase retrieval algorithm based on a wave propagation equation. In the new setup, the arrangement of the diffuser and the object is reversed. A plane wave incident on the diffuser generates a speckle field which, in turn, is used to illuminate the object. The transmitted wavefront is then directed to the camera sensor. The advantage of the proposed setup is the increased resolution since the limiting aperture is the full area of the sensor. [DOI: 10.2971/je0s.2009.09002]

Keywords: wavefront sensing, phase retrieval, image reconstruction techniques, speckle, diffraction theory, scattering, rough surfaces, optical sensing and sensors

\section{INTRODUCTION}

Speckle pattern is the random intensity distribution resulting when a coherent light is reflected or transmitted from a diffusing object [1]. Illumination of a test object with a speckle pattern is essential in the monitoring of biological activity [2], measurements of object roughness [3], thickness and range [4], and in microscopy [5]. All of these applications [2]-[5] use a fully-developed speckle field (FDSF). A speckle field is considered fully developed when the mean roughness height of the diffuser is in the order or larger than the light source wavelength $\lambda$ and the illuminated spot size is much larger than the any lateral scale of the diffuser [6]. Another application of FDSF illumination is in holography where the desired output is the complete wavefront from the illuminated object. In the reconstruction of a wavefront, a hologram made from a diffusely illuminated object can yield a reconstruction free of flaws resulting from dust particles and deficiencies in the optical system [7]. The applied diffuse illumination does not completely destroy the coherence of light which is essential for the wavefront reconstruction [7]. Current advances in optical sensor technology gave impetus to Digital Holography (DH) [8]. During recording in $\mathrm{DH}$, a test wavefront is superimposed with a physical reference beam and the resulting interference pattern is recorded (as digital hologram) using a camera. $\mathrm{Nu}$ merical reconstruction of the test wavefront is then carried out by simulating the diffraction of the conjugate of a digital reference beam on the digital hologram. One drawback of DH is the aberration introduced by the difference in the curvatures of the digital reference beam and the physical reference beam [9]. To compensate for this, additional phase factors have to be designed and tried out to completely remove this holographyinduced aberration. Completely avoiding this complication due to the reference beam would greatly simplify the wavefront analysis.

A parallel wavefront reconstruction method, which does not require a reference beam, is called phase retrieval where the numerical reconstruction is derived from the intensity variation of the object wave diffraction [10]. There are two general types of phase retrieval methods: 1) iterative; and 2) non iterative. In conventional iterative methods that utilize a single Fourier intensity measurement, quantization error due to the limited number of detector energy levels is found critical in the reconstruction of wavefronts with high-spatial frequency contents [11]. Another critical issue in iterative phase retrieval is called the stagnation problem where the calculation converges to a local minimum. To avoid the stagnation problem, various a priori knowledge or constraints are imposed such as object support area, and the object must be non-negative and real [11]. Such constraints, however, are restrictive to the choice of test object wavefronts that can be investigated. A method to partially reduce quantization error by enhancing the measurement of the single Fourier intensity using a technique called multispectral method is described in [11]. However, the main difficulty that leads to the quantization error in these methods still remains. The difficulty arises from the limited bit depth of detectors which is not able to efficiently capture the large dynamic range (maximum intensity level minus the minimum intensity level) of the Fourier intensity distribution that usually comes with an intensely bright focused spot [11]. The useful high frequency components that are located far from the intense focus will still be lost to the camera noise despite having to use expensive 12-bit detectors [11]. 
In noniterative phase retrieval or phase diversity methods, the paraxial form of the Helmholtz wave equation is used to analytically determine the test wavefront [12]. In these methods, the derivative of the wave intensity is derived from the difference of two defocused images and the small axial distance between the measurement planes [12]. At such close distances, the nominal intensity variation may not be sufficiently detected which is also attributed to quantization error. In addition, the paraxial condition limits the applications of these methods to test wavefronts that propagate near the optical axis. For test object wavefronts with high spatial frequencies and propagating at large angles, the paraxial condition is not satisfied, hence, the method cannot be readily used. For high numerical aperture (N.A.), the Rayleigh-Sommerfeld (RS) diffraction equation may be used since it can be implemented without the paraxial approximation [13]. To compound the effects of quantization error, iterative or noniterative methods which heavily rely on one or two intensity measurements are also susceptible to random errors due to intensity fluctuations and other perturbations.

A study by Pedrini et.al., [14] demonstrated wavefront reconstruction by utilizing 21 interferograms (object diffraction patterns) and an iterative algorithm based on the RS equation. The unique feature of this approach is the significant increase in the available physical intensity data which makes it robust to intensity fluctuation error and helps in avoiding the stagnation problem. The influence of quantization error is reduced attributed to the averaging effect of the multiple measurements resulting in higher signal to noise ratio. In addition, there is no need to impose various constraints such as object area of support or the object must be nonnegative and real. Hence, the technique is nonrestrictive with regard to the choice of samples. The technique was successfully demonstrated for the pure amplitude type object (amplitude mask) used [14]. In that study, it was noted that the limited dynamic range ( 8 bits) of the CCD used hampered the wavefront reconstruction [14]. It was suggested that the use of better cameras and more interferograms will improve the reconstructions [14]. The multiple intensity approach has been extended to test objects with very rough surfaces [15]. Instead of the regular diffraction patterns used in [14], speckle intensity measurements were input into the iterative phase retrieval algorithm [15]. Sub-pixel scanning [15] to enhance the speckle measurements and aperture synthesis [16] to increase the effective numerical aperture, were also demonstrated. The multiple intensity approach $[14,15]$ has also been adapted to reconstruct smooth or low-curvature wavefronts. The nominal intensity variation along the axial direction for smooth wavefronts may be perturbed by introducing an amplitude diffuser [17] or a phase diffuser [18]. Analysis of smooth wavefronts is a critical task in optical testing for aberrations. The test wavefront incident on the diffuser generates a speckle field which, in turn, is sampled at axially displaced planes and utilized in the phase retrieval. In [15], it is remarked that the plane wave illuminating the rough object surface already generates a speckled wavefront. For such test wavefronts, the introduction of a diffuser in the setup is not needed.

In this paper, as well as in $[17,18]$, the speckle field being utilized for the wavefront reconstruction is referred to as a partially-developed speckle field (PDSF). A speckle field is considered partially developed when the mean roughness height of the diffuser does not exceed $\lambda$ and the illuminated spot size is much larger than any lateral scale of the diffuser [6]. In [18], the test wavefront is made incident on an energy efficient phase diffuser which converts the test wavefront to a PDSF. At each of the measurement planes, the high frequency speckle intensity distribution exhibits a high dynamic range. Thus, the test wavefront, which is coherently transmitted in the speckle patterns, is efficiently sampled. The speckle statistics of a wavefront traversing the specific diffuser used is described in [18]. The nominal roughness height of the diffuser facilitates the transmittance of the scattered wave component and the unperturbed wave component (i.e., PDSF). Compared to interferometry/holography-based methods [9], the phase retrieval method offers a simple construction of the setup and straightforward aberration correction since no reference beam is utilized $[19,20]$. Compared to the conventional single Fourier intensity [11] phase retrieval methods, the speckle patterns that carry the complete wavefront information have large dynamic ranges, hence, the inexpensive 8-bit camera used is found to be sufficient. In addition, the use of multiple speckle intensities, compared to a single Fourier intensity or two defocused images, provides more physical data for the algorithm and facilitates robustness to intensity fluctuations. The speckle intensity also varies significantly between the measurement planes which aids in avoiding the stagnation problem without the need for additional constraints. Compared to noniterative methods based on the paraxial Helmholtz equation [12], the speckle-based method uses the RS equation which is suitable for a high N.A. system and for object wavefronts with high spatial frequencies. Compared to the regular diffraction patterns being utilized in [14], the speckle intensity distributions formed by a wide range of spatial frequencies have high dynamic ranges. Hence, the speckle-based method is not seriously affected by the quantization error associated with the convenient 8-bit cameras. The significant increase in the reliable intensity data also means faster convergence of the phase calculation. The accuracy of this speckle-based phase retrieval technique has been established to be comparable with conventional interferometry [21, 22]. In [21], the interferograms resulting from the retrieved phase difference between tilted wavefronts were correlated to the physical rotation of the prism through which the wavefront passes. In [22], interferograms of surface deformations due to thermal loading were analyzed. Traditionally, interference pattern-based metrology has been carried out using interferometric/holographic methods. The retrieved phase interferograms in [21, 22] are analogous to double-exposure holographic interferograms generated upon subtraction of reconstructed phases obtained via DH [8]. The utilization of retrieved phase interferograms in interferometric metrology in [21, 22] and the aberration analysis in [19] are considered small steps toward the convergence and unification of the two parallel coherent wavefront reconstruction methods: interferometry/holography, on one hand; and phase retrieval, on the other.

In the previous related studies on wavefront sensing, a plane wave illuminated a phase object (lens/prism) [17, 18, 20, 21, 22 ] and the transmitted wavefront is made incident on a dif- 
fuser. Also in [22], a plane wave illuminated a Plexiglas surface and the reflected wavefront is incident to a diffuser before detection. For the particular wavefronts tested, the performance of the reconstruction technique in terms of phasefront resolution is found satisfactory. In the method employed in the previous studies, which will be referred to as the conventional method, an iris aperture attached to the diffuser is utilized to set the speckle size for efficient sampling at the detector planes. The small iris aperture, however, limits the system resolution which needs to be maximized especially if the test object wavefront has high spatial frequencies.

The main motivation of this study is to reconstruct object wavefronts with high spatial frequencies using a novel setup for the speckle based phase retrieval technique. In the new speckle illumination setup, a plane wave is made incident on the phase diffuser to generate a PDSF. The PDSF, in turn, is used to illuminate the various object specimens being investigated. The transmitted wave from a specimen is then directed to the camera sensor (without any intervening optical elements like an iris aperture) for sampling. Compared to the conventional method [18], the limiting aperture in the proposed technique is the full sensing area of the photodetector, hence, in principle, has higher resolution. Also in the present study, the performances of the different multiple intensity methods (multiple diffraction method [14], conventional speckle-based method [18] and the proposed speckle illumination method) in the reconstructions of wavefronts with high spatial frequencies are investigated.

\section{BASIC PRINCIPLES}

The mechanism for wavefront reconstruction using speckle illumination is the modulation of the PDSF by the optical properties of the specimen. The amplitude and phase structures of the specimen influence both the plane wave and scattered wave components of the PDSF [18]. If the specimen is not highly scattering, the transmitted light is still a PDSF. The transmitted PDSF, bearing complete information of the object wave, is then sampled using a photodetector at axially displaced planes. Using the speckle intensity measurements, the complete wavefront is reconstructed by an iterative use of the angular spectrum method of solving the RS wave propagation equation. The sampled speckle patterns are represented as $m \times n$ matrices and then are sent into the RS equation [14], which in discrete form is given by

$$
\begin{aligned}
& U_{p+1}\left(k \Delta x^{\prime}, l \Delta y^{\prime}, z\right)= \\
& \Im^{-1}\left(\Im\left\{\left[I_{p}(m \Delta x, n \Delta y)\right]^{1 / 2} \exp \left[i \phi_{p}(m \Delta x, n \Delta y)\right]\right\}\right. \\
& \left.\quad \times \exp \left[(-i 2 \pi z / \lambda)\left(1-\frac{\lambda^{2} m^{2}}{\Delta x^{2}}-\frac{\lambda^{2} n^{2}}{\Delta y^{2}}\right)^{1 / 2}\right]\right)
\end{aligned}
$$

where $U_{p+1}$ is the complex amplitude calculated at the $(p+1)$ plane, $p=1 \ldots 19 ;\left(k \Delta x^{\prime}, l \Delta y^{\prime}\right)$ and $(m \Delta x, n \Delta y)$ are the coordinates at the $(p+1)$ and $p$ planes, respectively; $\Im$ indicates 2D Fourier transformation; $I_{p}$ is the speckle intensity distribution at $p$ plane; and $\Delta x \times \Delta y$ are the dimensions of the camera pixels. The phase (initially a guessed random phase) is extracted from the calculated complex amplitude, $\phi=\tan ^{-1}[\operatorname{Im}(U) / \operatorname{Re}(U)]$. The successive propagation between the measurement planes is iterated to enhance the reconstruction. Upon reconstruction, the wavefront can then be propagated at specific specimen planes to characterize its intensity and phase structures. The intensity is obtained from the square of the absolute value of the complex amplitude and the phase is derived from the angle of the complex-valued wavefront. To display the desired wavefront phase, the reconstructed phase is numerically filtered to remove the rapidly varying phase of the scattered wave component of the PDSF. It is noted that the low-pass filtering of the speckle phase with a suitable filter size does not decrease the system resolution but, mainly, enhances the display of the desired wavefront phase. Intensity of reconstruction may be brought into focus and can be utilized in the localization of planes of interest within a volume specimen. Phase distribution for a curved or tilted wavefront is represented by a fringe pattern where the bright (or dark) bands indicate regions of constant phase with values wrapped in $2 \pi$. Further discussions on the principles of the algorithm may be found in the cited related works.

The spatial resolution of the reconstruction of object wavefronts with high spatial frequencies using the proposed setup is similar to that in $\mathrm{DH}$. During recording, both the proposed technique and DH do not use a physical aperture [8]. During the numerical reconstruction, both methods use wave propagation from the measurement/hologram plane to an observation/object plane [8]. In DH, the hologram is the aperture of the optical system with side length $N \Delta x$, where $N$ is the number of pixels and $\Delta x$ is the pixel size along $x$-direction [8]. The diffraction limited resolution of the reconstructed wavefront, in free space, is given by $\lambda /$ N.A., where N.A. $=N \Delta x / z$ is the effective numerical aperture, with $z$ being the object to sensor distance [8]. Similar expression for resolution applies to the proposed speckle-based technique. The recorded speckle pattern is the aperture of the optical system with side length $N \Delta x$. For the case of the conventional method applied to test object wavefronts with high spatial frequencies, the diameter of the iris aperture is used in place of $N \Delta x$. In this study, various types of transmitting objects that introduce small perturbations on the PDSF are considered. Pure amplitude objects are characterized by complete or partial absorption of light intensity. Pure phase objects do not appreciably absorb light but changes the wavefront phase of the incident light. When light travels through a phase object, the phase of transmitted light is shifted by an amount proportional to the optical path (refractive index $\times$ thickness) traveled within the object.

\section{EXPERIMENTS}

Figure 1 shows the schematic diagram for the speckle illumination of a specimen and generation of speckle intensity patterns $\left(I_{1}, I_{2} \ldots, I_{20}\right)$ to be recorded. The laser beam (HeNe laser, $\lambda=633 \mathrm{~nm}$ ) incident on a phase diffuser generates a PDSF [18]. The speckle field illuminates the specimen which, in turn, further scatters the field. The mean speckle size at the specimen plane is set by the diffuser aperture (diameter, $\varnothing=1.7 \mathrm{~mm}), \lambda$ and the distance from the aperture to specimen. The distance from diffuser aperture to specimen is $20 \mathrm{~mm}$. 


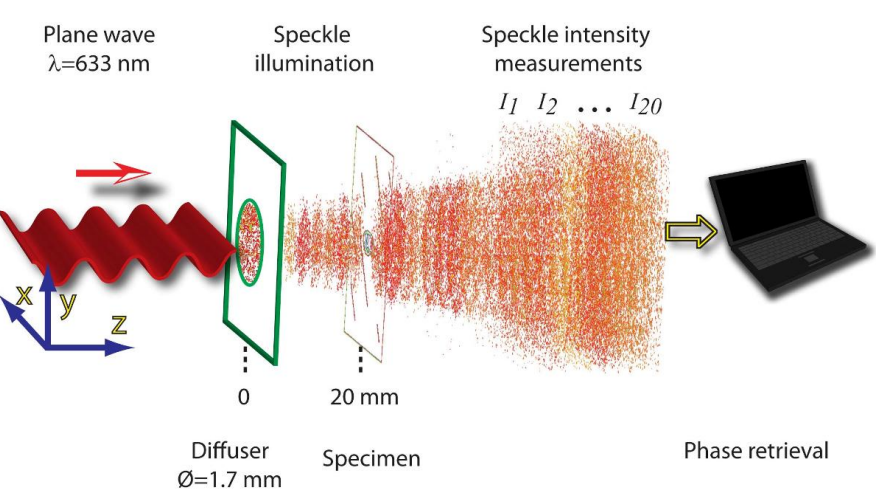

FIG. 1 Schematic diagram of the setup.

The phase diffuser is the key element of the setup. The following summarize the experimental conditions during its fabrication [18]. The photolithographic process is carried out in a clean room environment. The photoresist (S1805) is spun onto the glass plate to a thickness of $500 \mathrm{~nm}$. To ensure the linear response of the photoresist with respect to intensity, the plate is pre-illuminated for $2 \mathrm{sec}$ in ultraviolet light. The photoresist is exposed to a fully-developed speckle field (mean transverse speckle size, $10 \mu \mathrm{m}$ ) generated when a laser beam (Helium Cadmium, $\lambda=442 \mathrm{~nm}$, max. power of $300 \mathrm{~mW}$ ) is incident on a ground glass diffuser. The photoresist plate is then chemically developed, rinsed and dried to produce the phase diffuser. Photoresist, as the phase diffusing medium, was chosen since the maximum roughness height can be controlled by varying the exposure time. Speckle projection was chosen as the mode for etching the photoresist since the mean transverse size of the projected speckles and, in turn, that of the phase diffuser indentations, can be controlled.

For this study, specimens with various amplitude and phase distributions are investigated. The speckle patterns transmitted from the specimen are measured sequentially at $N=20$ equal interval planes using a CMOS camera (Opticstar PL$130 \mathrm{M}, 1280(\mathrm{H}) \times 1024(\mathrm{~V})$ pixels, pixel dimensions: $5.2 \mu \mathrm{m} \times$ $5.2 \mu \mathrm{m})$ mounted on a motorized stage. The distance from the specimen to the first measurement plane is about $10 \mathrm{~mm}$ $(\mathrm{N} . \mathrm{A} .=0.263)$ and the sequential measurement distance $(\Delta z)$ is $1 \mathrm{~mm}$. The sampling period to complete 20 cycles of speckle recording and moving the camera in the measurement planes is about 1 minute. For phase retrieval, the speckle intensity patterns are input into the computer algorithm described in Section 2 of this paper and in [18].

\section{RESULTS AND DISCUSSION}

Figure 2(a) shows the intensity (left image) and filtered phase (right image) of the reconstructed wavefront through the diffuser without any specimen. This wavefront reconstruction serves as a control to compare qualitatively the results when specimens are placed in the setup. The planar phasefront is the unperturbed wavefront phase component of the PDSF transmitted by the diffuser. It is remarked that a quantitative analysis using Zernike polynomials and Strehl ratios [19] and extended Nijboer Zernike vector diffraction theory [23] may be carried out to determine the nature and extent and also

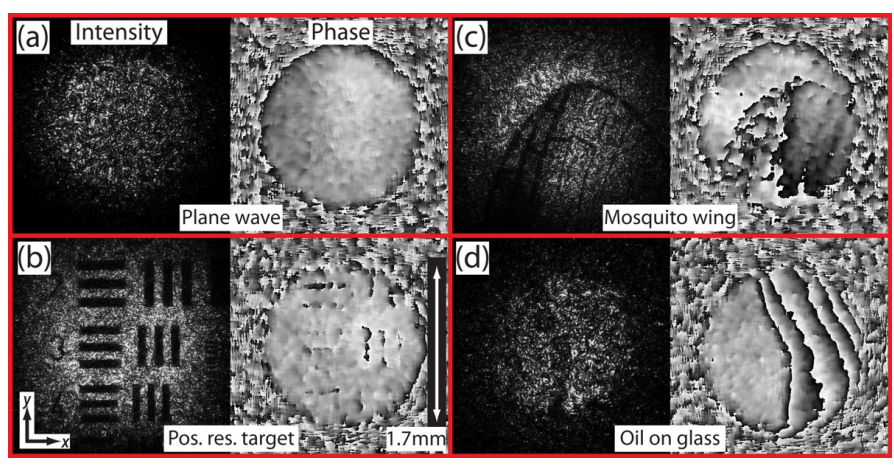

FIC. 2 Complete wavefront reconstructions: intensity (left image) and filtered phase (right image) for various types of object specimens. (a) Control (plane wave illumination). (b) Pure amplitude (positive resolution target). (c) Amplitude-phase (mosquito wing with venation). (d) Pure phase (lubricating oil spread on the right side of a glass slide). (Movie of wavefront propagation, videosize: $1.6 \mathrm{MB}$, format: mov, see Fig2.mov).

to numerically correct for the phase errors. Figures 2(b)-2(d) are the experimental results for the different types of specimens investigated. Figure 2(b) shows the reconstructed intensity and phase of a positive resolution target (chrome pattern on clear background, IMT) which is considered to be a pure amplitude-type specimen. The phase is nearly constant in the areas outside the parallel bars. It is noted that the target glass plate oriented parallel to the diffuser phase plate did not introduce any significant phase shift. The intensity plot reveals the speckle-illuminated portion of the target. For imaging applications where intensity distribution is the final output, this undesired speckle effect may be reduced by averaging several sets of reconstructions. Speckle reduction may also be carried out by space and wavelength diversity [24]. Shown in Figures 2(c) and 2(d) are the reconstructions for the specimens that are considered as semi-transparent due to the partial absorbance of the incident beam. In Figure 2(c), the specimen shown is a portion of a mosquito wing. The darker portion of the intensity plot on the left side is attributed to a thicker wing venation and higher absorbance of the incident light. For the same portion of the wing, the phase plot reveals some characteristic phase structures. Lighter portion in the intensity plot on the right side which covers a broader area indicates thinner wing venation and less light absorbance. For the same portion of the wing, the phase exhibits a gradient along the vertical direction. Two interpretations may be inferred: 1) The wing's thickness is tapering towards the edge, assuming a uniform refractive index; or, 2) The refractive index is spatially varying, assuming even thickness. Since only an approximate average wing thickness of less than 5 microns was obtained using a Vernier caliper, the exact interpretation may be established upon further validation.

In Figure 2(d), the specimen used is lubricating oil spread thinly on a microscope glass slide. For demonstration purposes, the illuminated area is the boundary between sections with oil and without oil. The intensity plot shows a faint trace of the interface of the two sections. The phase plot evidently reveals the distinction between the two sections. On the right hand portion, where the oil is spread, fringe contours indicate a gradual decrease in the oil thickness. The glass slide oriented parallel to the diffuser phase plate also did not in- 
troduce any significant phase shift. With a refractive index of about 1.47 for lubricating oils, a sample calculation for oil layer thickness per fringe is $\lambda / 1.47=0.43 \mu \mathrm{m}$. It is noted that if the oil were uniformly spread with a single height, the fringe pattern would have exhibited a single gray level indicating a uniform phase shift. As a further note on the choice of samples, the technique relies on the detection of partially developed speckles upon modulation by the object properties. If the wave transmitted through an optically thick sample (like a biological tissue) does not exhibit partially developed speckles, the present setup cannot be used. A movie file displays the propagation of the reconstructed wavefront. The importance of this video rendering is the enhanced visualization wherein the phase and intensity components of the complete wavefront are shown side by side. Propagation distance between successive frames is $\lambda / 8$ or $0.079 \mu \mathrm{m}$.

The performances of the three different modes of the multiple intensity approach in reconstructing high spatial frequency wavefronts are investigated. Figure 3 shows the three setups considered: 1) plane wave - object - camera (Figure 3(a), setup without diffuser, [14]); 2) plane wave - object - diffuser aperture - camera (Figure 3(b), conventional setup, [18]); and 3) plane wave - diffuser aperture - object - camera (Figure 3(c), proposed speckle illumination setup). To evaluate the quality of wavefront reconstruction, separate experiments for the intensity and phase are carried out. For the wavefront intensity, a negative mask resolution target (clear pattern of a chrome background, IMT) is used and considered as a pure amplitude type object. For the wavefront phase, a positive photoresist resolution target (photoresist relief pattern on a flat background) is used and considered as a pure phase type object. For each of the experiments, good reconstruction is achieved when the edges of the equally-spaced bars in the object wavefront reconstruction are well defined. In this comparative study, $N=20,10$ iterations and $\Delta z=1 \mathrm{~mm}$ are maintained for each of the three setups. In Figure 3, only 3 intensity measurements are depicted for simplicity.
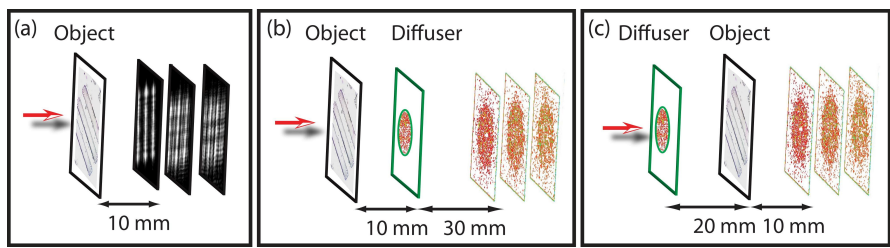

FIG. 3 Various setups for multiple-intensity phase retrieval (PR): (a) multiple diffraction pattern PR; (b) conventional setup for speckle-based PR; and, (c) proposed speckle illumination speckle-based PR.

The following experimental conditions for the distances are set so that comparisons can be made with the proposed technique. In the setup without diffuser, a plane wave illuminates the object specimen which is positioned $10 \mathrm{~mm}$ from the first measurement plane (N.A. $=0.263$ ). In the conventional setup, the distance from the specimen to diffuser aperture is $10 \mathrm{~mm}$ (N.A. $=0.085)$ and the distance from diffuser aperture to the first measurement plane is $30 \mathrm{~mm}$. In the speckle illumination setup, the distance from diffuser aperture to specimen is $20 \mathrm{~mm}$ and the distance from specimen to the first measurement plane is $10 \mathrm{~mm}$ (N.A. $=0.263)$.

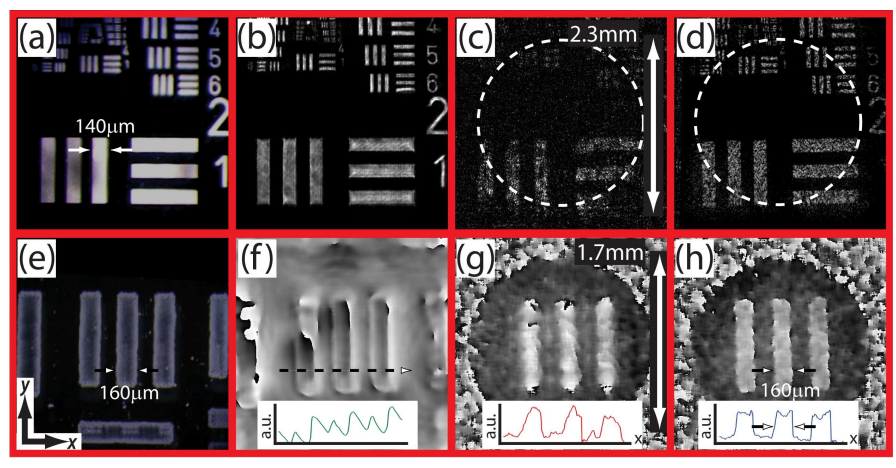

FIG. 4 (a) Negative mask resolution target (pure amplitude type object) and the reconstructed intensities for various setups (b-d). (e) Positive photoresist resolution target and the reconstructed phases for the various setups (f-h).

Figure 4(a) shows a photographic image of a portion of the negative mask resolution target. A reconstructed wavefront is expected to exhibit an intensity distribution, at the object plane, bearing similar parallel bars. However, it is remarked that for the 2 speckle-based methods, the expected intensity distribution inside the transmitting bars is modulated by speckles due to the introduction of the diffuser. Outside the bars, on the other hand, there should be no transmitted intensity distribution and speckles. Figure 4(b) shows the reconstructed intensity for the setup without diffuser. The well defined edges of the parallel bars indicate wavefront reconstruction. For the conventional setup (Figure 4(c)), the edges of the bars are not resolved and the region outside the bars is degraded with speckle noise. The poor overall quality is due to the small iris aperture (indicated by the circular area) of the diffuser used. The small aperture was not able to capture the high frequencies of the object wavefront. Figure 4(d) shows the reconstructed intensity using the speckle illumination setup. Compared to Figure 4(c), the edges of the bars are well resolved and the speckle noise outside the bars is nominal. The significant improvement in the quality of reconstruction is attributed to increases in effective aperture size and N.A. In Figure 4(d), the observed speckles inside the transmitting bars are due to the diffuser, as expected. With the given the experimental conditions and the negative mask target used, the setup without diffuser (multiple diffraction method) and speckle illumination setup both yield good reconstructed intensities. For the speckle illumination setup, speckles inside the parallel bars may be reduced by speckle averaging techniques as mentioned earlier in the discussion of Figure 2(b).

Figure 4(e) shows a photographic image of a portion of the positive photoresist target. The details of target exposed to ultraviolet light, during its fabrication, become soluble to the photoresist developer leaving a relief image of the target. The thickness of the photoresist is $1.95 \mu \mathrm{m}$ which is measured using a Dektak profilometer. The refractive index of the photoresist (S1805) at $633 \mathrm{~nm}$ is about 1.65. Figure 4(f) shows the phase reconstruction when regular diffraction patterns are used in the phase retrieval. The faint traces of the parallel bars with poor background contrast may be considered a poor reconstruction. This is attributed to the insignificant intensity variation between the axially displaced diffraction patterns when the pure phase type object used. It is noted that in the case of the negative mask target (Figure 4(a)), the opaque edges of 
the submillimeter-size target bars resulted in diffraction patterns that vary significantly between the measurement planes. For the transparent photoresist target, enhanced diffracted intensity variation is facilitated by the use of the phase diffuser which generates a PDSF. Using the conventional setup, the contrast between the parallel bars and the background (Figure 4(g)) is increased compared to Figure 4(f). This is attributed to the significant speckle intensity variation between the measurement planes which aids in the phase retrieval. However, the edges of the bars are still not well resolved. This is attributed to the small iris aperture and N.A. Using the proposed setup, the edges of the parallel bars are well resolved and the contrast is more enhanced (Figure 4(h)). The corresponding line scans of the phase fronts show that the sharpest transition between the phase bars and background and correct bar width are evident in Figure 4(h). For the given experimental conditions and the particular pure phase object used, the proposed speckle technique yields the most enhanced phase reconstruction.

One of the benefits of wavefront reconstruction is the resolution of contiguous planes of three-dimensional (3D) specimens by numerical focusing. When a wavefront is brought into focus at a particular plane, this plane appears to be sharp whereas the other planes are blurred. To demonstrate this attribute of the technique, two optical fiber tips axially separated by $2 \mathrm{~mm}$ are illuminated with speckled light. The transmitted light is then sampled and input into the phase retrieval algorithm to reconstruct the wavefront. Figure 5 shows the intensities of the reconstruction at different axial positions (1 mm increment): (a) foreground, (b) location of the first fiber tip, (c) between the two fiber tips, (d) location of the second fiber tip, and (e) background. The outlines of each of the fiber tips and traces of line-focused intensity are revealed at their respective axial locations demonstrating 3D localization (Figures 5(b) and 5(d)). The phase plot (Figure 5(f)) shows two narrow vertical strips that are shifted with respect to the nearly constant background phase. A movie file shows the intensities of the reconstructed wavefront by numerical focusing at different axial positions demonstrating 3D localization. Movie rendering of the wavefront focusing gives a visual account of

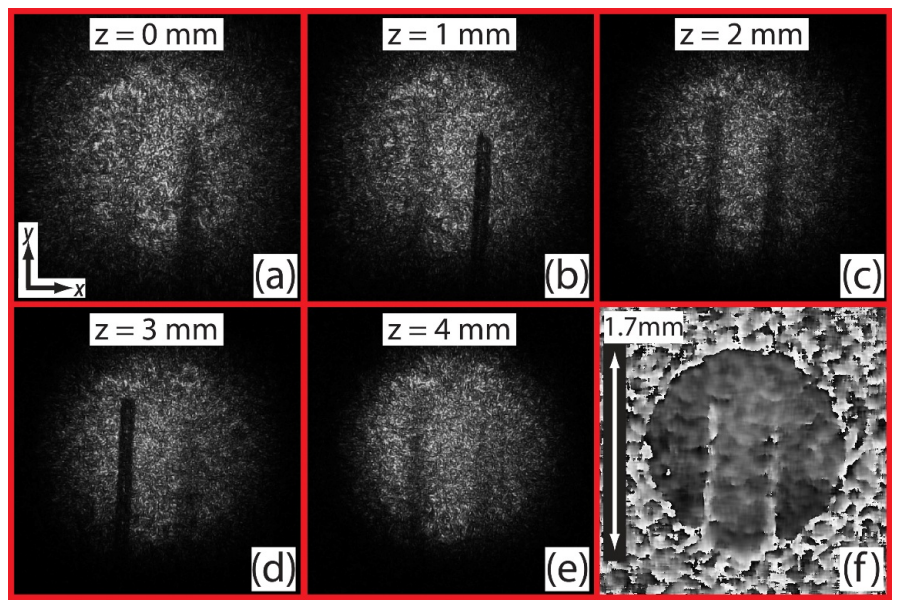

FIG. 5 (a)-(e) Intensities at various planes demonstrating 3D localization of 2 glass fibers (axially separated by $2 \mathrm{~mm}$ ). (f) Phase at center plane. (Movie depicts $3 \mathrm{D}$ localization, videosize: 1.3 MB, format: mov, see Fig5.mov). the exact locations of different objects in a volume space. It is remarked that similar movies are also found in digital holographic studies but, to our knowledge, have not been utilized in any phase retrieval studies. The propagation distance between successive frames is $\Delta z=0.5 \mathrm{~mm}$.

\section{CONCLUSIONS}

A novel setup for the speckle-based phase retrieval method by illuminating the object with a PDSF is proposed. The properties of the specimen are impressed upon the plane wave component of the incident PDSF which, then, are coherently transmitted in the sampled speckle patterns. Complete wavefront reconstruction using the sampled speckles and an iterative algorithm based on RS equation is demonstrated. The proposed setup for the speckle-based phase retrieval method offers increased resolution compared to the conventional setup. The high frequency wavefront components from the object specimen propagate at a large angle. In the conventional setup, the diffuser aperture filters out the high frequency components of the object wavefront which adversely affect the information gathering capability of the system. The interest in using the proposed speckle illumination setup is to maximize the resolution of the reconstruction since, in this technique, the limiting aperture is the whole detector sensing area.

\section{ACKNOWLEDGEMENTS}

The authors greatly acknowledge the financial support from the Danish Council for Technology and Innovation under the Innovation Consortium CINO (Centre for Industrial Nano Optics). The authors also thank Henrik Chresten Pedersen and Jørgen Stubager for their kind assistance in the preparation of the photoresist plates. P. Almoro acknowledges the University of the Philippines - Office of the Vice-Chancellor for Research and Development for the financial support.

\section{References}

[1] J. Goodman, Statistical optics (Wiley, 1985).

[2] M. Pajuelo, G. Baldwin, H. Rabal, N. Cap, R. Arizaga, and M. Trivi, "Bio-speckle assessment of bruising in fruits" Opt. Laser. Eng. 40, 13-24 (2003).

[3] J. Garcia, Z. Zalevsky, P. Garcia-Martinez, C. Ferreira, M. Teicher, and $\mathrm{Y}$. Beiderman, "Three-dimensional mapping and range measurement by means of projected speckle patterns" Appl. Optics. 47, 3032-3040 (2008).

[4] P. Lehmann, "Surface-roughness measurement based on the intensity correlation function of scattered light under specklepattern illumination" Appl. Optics. 38, 1144-1152 (1999).

[5] D. Lim, K. K. Chu, and J. Mertz, “Wide-field fluorescence sectioning with hybrid speckle and uniform-illumination microscopy" Opt. Lett. 33, 1819-1821 (2008).

[6] W. Wang, S. G. Hanson, and M. Takeda, "Complex amplitude correlations of dynamic laser speckle in complex ABCD optical systems" J. Opt. Soc. Am. A. 23, 2198-2207 (2006). 
[7] E. N. Leith and J. Upatnieks, "Wavefront reconstruction with diffused illumination and three-dimensional objects" J. Opt. Soc. Am. 54, 1295 (1964).

[8] U. Schnars and W. Juptner, "Digital recording and numerical reconstruction of holograms" Meas. Sci. Technol. 13, R85-R101 (2002).

[9] G. Pedrini, S. Schedin, and H. J. Tiziani, "Aberration compensation in digital holographic reconstruction of microscopic objects" J. Mod. Optic. 48, 1035-1041 (2001).

[10] J. R. Fienup, “Phase retrieval algorithms: a comparison” Appl. Optics. 21, 2758-2769 (1982).

[11] S. Yang and H. Takajo, "Quantization error reduction in the measurement of Fourier intensity for phase retrieval" Jpn. J. Appl. Phys. 45, 1832-1835 (2006).

[12] M. R. Teague, “Deterministic phase retrieval: a Green's function solution" J. Opt. Soc. Am. 73, 1434-1441 (1983).

[13] J. Goodman, Introduction to Fourier Optics (Roberts and Company, 2005).

[14] G. Pedrini, W. Osten, and Y. Zhang, "Wave-front reconstruction from a sequence of interferograms recorded at different planes" Opt. Lett. 30, 833-835 (2005).

[15] P. Almoro, G. Pedrini, and W. Osten, "Complete wavefront reconstruction using sequential intensity measurements of a volume speckle field" Appl. Optics. 45, 8596-8605 (2006).

[16] P. Almoro, G. Pedrini, and W. Osten, "Aperture synthesis in phase retrieval using a volume-speckle field" Opt. Lett. 32, 733-735
(2007).

[17] A. Anand, G. Pedrini, W. Osten, and P. Almoro, "Wavefront sensing with random amplitude mask and phase retrieval" opt. Lett. 32, 1584-1586 (2007).

[18] P. F. Almoro and S. G. Hanson, "Random phase plate for wavefront sensing via phase retrieval and a volume speckle field" Appl. Optics. 47, 2979-2987 (2008).

[19] P. F. Almoro, P. N. Gundu, and S. G. Hanson, "Numerical correction of aberrations via phase retrieval with speckle illumination." in preparation, (2009).

[20] P. F. Almoro and S. G. Hanson, "Wavefront sensing using speckles with fringe compensation" Opt. Express 16, 7608-7618 (2008).

[21] P. F. Almoro, G. Pedrini, A. Anand, W. Osten, and S. G. Hanson, "Interferometric evaluation of angular displacements using phase retrieval” Opt. Lett. 33, 2041-2043 (2008).

[22] P. F. Almoro, G. Pedrini, A. Anand, W. Osten, and S.G. Hanson, "Angular displacement and deformation analyses using a specklebased wavefront sensor" in preparation, (2009).

[23] S. van Haver, J.J.M. Braat, P. Dirksen, A.J.E.M. Janssen, "HighNA aberration retrieval with the extended Nijboer-Zernike vector diffraction theory" J. Europ. Opt. Soc. Rap. Public. 1, 06004 (2006).

[24] C. Saloma, S. Kawata, and S. Minami, "A laser diode microscope that generates weakly speckled images" Opt. Lett. 15, 203-205 (1990). 